\title{
DIREITO À SAÚDE NO DISCURSO DO PODER JUDICIÁRIO E DO PODER PÚBLICO: ANÁLISE DE DECISÃO JUDICIAL*
}

\author{
Maiara Mariottoํ, Laura Filomena Santos de Araújo², Roseney Bellato²,
} Janderléia Valéria Dolina ${ }^{4}$

\begin{abstract}
RESUMO: Estudo objetivou analisar a contraposição entre os discursos do Poder Judiciário e do Poder Executivo, contendores em decisão judicial acerca do direito à saúde de criança em situação de adoecimento crônico com desnutrição grave. Trata-se de estudo descritivo de abordagem qualitativa, baseado em análise documental de decisão judicial do Tribunal de Justiça de Mato Grosso, demandando o fornecimento de insumo alimentar e medicamentos. A análise mostrou que o Estado, apesar de suas responsabilidades em garanti-los, nega concedê-los afirmando a necessidade de gestão na distribuição farmacêutica, o desconhecimento do risco de morte do bebê e limites nos recursos financeiros da saúde. O estudo evidencia a contradição do discurso do Estado brasileiro na proteção de seus cidadãos, especificamente as crianças. Esta contradição mostra que o Estado descumpre abertamente a legislação, considerando as questões administrativas como mais prioritárias que o bem estar de criança vulnerável. DESCRITORES: Direito à saúde; Decisões judiciais; Instituições de saúde; Saúde da criança.

\section{THE RIGHT TO HEALTH IN THE DISCOURSE OF THE JUDICIARY AND THE PUBLIC AUTHORITIES: ANALYSIS OF JUDICIAL DECISION-MAKING}

\begin{abstract}
This study aimed to analyze the contrast between the discourses of the Judiciary and the Public Authority, contenders in judicial decision-making regarding the right to health of the child in a situation of chronic illness with serious malnutrition. It is a descriptive study with a qualitative approach, based on documental analysis of the judicial decision-making of the Court of Justice of Mato Grosso, requiring the provision of nutritional intake and medications. The analysis showed that the State, in spite of its responsibilities to guarantee these, declines to concede them, asserting the need for management in the distribution of medications, lack of knowledge of the risk of death for the baby, and limits on the financial resources for health. The study evidences the contradiction of the discourse of the Brazilian State in the protection of its citizens, in particular the children. This contradiction shows that the State openly fails to comply with the legislation, considering administrative issues as having priority over the well-being of vulnerable children. DESCRIPTORS: Right to health; Judicial decisions; Health institutions; Children's health.

\section{DERECHO A LA SALUD EN EL DISCURSO DEL PODER JUDICIARIO Y DEL PODER PÚBLICO: ANÁLISIS DE DECISIÓN JUDICIAL}

RESUMEN: Estudio cuya finalidad fue analizar la contraposición entre los discursos del Poder Judiciario y del Poder Ejecutivo, responsables por decisión judicial acerca del derecho a la salud del niño en situación de enfermedad crónica con desnutrición grave. Se trata de estudio descriptivo de abordaje cualitativo, basado en análisis documental de decisión judicial del Tribunal de Justicia de Mato Grosso, demandando fornecimiento de insumo alimentar y medicamentos. El análisis evidenció que el Estado, a pesar de sus responsabilidades en garantizarlos, niega concederlos afirmando la necesidad de gestión en la distribución farmacéutica, el desconocimiento del riesgo de muerte del bebé y límites en los recursos financieros de la salud. El estudio evidencia la contradicción del discurso del Estado brasileño en la protección de sus ciudadanos, específicamente los niños. Esta contradicción muestra que el Estado no cumple la legislación, dando más prioridad a las cuestiones administrativas que al bienestar del niño vulnerable. DESCRIPTORES: Derecho a la salud; Decisiones judiciales; Instituciones de salud; Salud del niño.

\footnotetext{
*Estudo vinculado à pesquisa matricial “As instituições de saúde e do poder judiciário como mediadores na efetivação do direito pátrio em saúde: análise de itinerários terapêuticos de pessoas/famílias no SUS/MT” (Pesquisa DITSUS), sob responsabilidade do Grupo de Pesquisa Enfermagem, Saúde e Cidadania -GPESC.

${ }^{1}$ Graduanda da Faculdade de Enfermagem da Universidade Federal do Mato Grosso - FAEN UFMT. Bolsista de Iniciação Científica pela Fundação de Amparo à Pesquisa do Estado de Mato Grosso. Membro do GPESC.

${ }^{2}$ Enfermeira. Doutora em Enfermagem. Professora da FAEN UFMT. Líder do GPESC.

${ }^{3}$ Enfermeira. Doutora em Enfermagem. Professora da FAEN UFMT. Membro do GPESC.

${ }^{4}$ Enfermeira. Mestre em Enfermagem. Membro do GPESC.
} 


\section{INTRODUÇÃO}

A saúde é um direito garantido desde 1988 através da Constituição Federal Brasileira que, em seu artigo 196, preconiza ser um direito social fundamental de caráter universal que deve ser assegurado pelo Estado e efetivado por intermédio de políticas sociais e econômicas ${ }^{(1)}$. Para a efetivação deste direito constitucional, em 1990 foi promulgada a Lei 8.080 que instituiu o Sistema Único de Saúde (SUS), norteado pelos Princípios de Universalidade, Integralidade e Equidade ${ }^{(2)}$.

Dentre os princípios norteadores do SUS, a Universalidade trouxe grande avanço à garantia do direito à saúde, como por exemplo, o acesso gratuito aos tratamentos, dentre estes os medicamentos e insumos. Diante disto, em 2001, o Ministério da Saúde (MS) dispôs a Política Nacional de Medicamentos (PNM) instituindo em 2003, o Departamento de Assistência Farmacêutica e Insumos Estratégicos visando normatizar, promover e coordenar a organização da assistência farmacêutica nos diferentes níveis da atenção à saúde ${ }^{(3)}$.

A PNM tem como propósito garantir a segurança, a eficácia e a qualidade dos medicamentos, além de promover o uso racional e o acesso da população àqueles classificados como essenciais. Nesse sentido, a Relação Nacional de Medicamentos Essenciais (RENAME) elenca aqueles destinados aos agravos que mais acometem a população brasileira ${ }^{(3)}$, bem como os medicamentos excepcionais, que são de elevado valor unitário, ou que requerem uso contínuo, o que os torna excessivamente $\operatorname{caros}^{(4)}$.

Dentro do contexto da universalidade dos tratamentos, a alimentação e a nutrição, considerados requisitos básicos para o desenvolvimento e crescimento saudável com qualidade de vida e cidadania, passaram a fazer parte do rol de direitos do cidadão. Em 2011, o MS instituiu a Política Nacional de Alimentação e Nutrição estabelecendo que os órgãos estaduais deveriam destinar recursos para as ações desse campo na rede de atenção à saúde dentro de sua abrangência ${ }^{(5)}$.

As políticas apresentadas mostram que, segundo os textos legais, o direito a saúde está constitucionalmente garantido e deveria haver em nosso país um sistema de saúde universal, norteado pelos princípios da integralidade e equidade, com políticas e ações que deveriam atender as necessidades de toda população. No entanto, deparamo-nos com uma realidade que tem se mostrado contraditória, pois os cidadãos necessitam buscar, por via judicial, a garantia deste direito. Essa busca pelo direito à saúde, por meio do poder judiciário, tem sido denominada como o fenômeno da judicialização da saúde $^{(6)}$, traduzido como garantia de acesso a bens e serviços de saúde por intermédio do recurso a ações judiciais.

Tal fenômeno sugere maior politização dos cidadãos em busca da concretização dos seus direitos, ainda que judicialmente. No entanto, percebe-se que as ações movidas nesse âmbito, em sua maioria, são para fornecimento de medicamentos ou insumos, mostrando que as muitas demandas pontuais da população no campo da saúde, embora já garantidas legalmente, não são atendidas pelo Estado ${ }^{(7)}$. Portanto, a intervenção judicial tem se mostrado pouco capaz de produzir modificações efetivas no campo da saúde, uma vez que as ações individuais não tomam o cunho coletivo, de modo a determinar a reorganização dos serviços de saúde e sua oferta de cuidado ${ }^{(7)}$.

Estudos realizados e que fazem parte de uma pesquisa matricial, na qual está inserido este trabalho, têm mostrado que, mesmo populações amparadas por Estatutos e Leis específicas, como crianças e idosos, ainda encontram barreiras para acesso aos medicamentos e insumos no $\mathrm{SUS}^{(8-10)}$. No caso de crianças, além da Constituição Federal, o Estatuto da Criança e do Adolescente (ECA) lhes garante o direito de proteção à vida e à saúde, mediante a efetivação de políticas sociais públicas que permitam um saudável nascimento e desenvolvimento ${ }^{(11)}$.

Este estudo pode contribuir com a efetivação do Sistema Único de Saúde, pois permite evidenciar a não implementação efetiva das políticas de proteção à criança, ainda que esta esteja em situação de vulnerabilidade inconteste. Entende-se que esta não efetivação programática pode prejudicar a integralidade no cuidado à criança, que pode ser conceitualizada como a garantia do uso dos cidadãos dos diferentes recursos tecnológicos de acordo com suas condições clínicas e epidemiológicas $^{(12)}$. Além disso, o cuidado infantil deve ser compreendido como um projeto que demanda a intersetorialidade pelo envolvimento de vários órgãos da sociedade civil e do Estado na resolução dos problemas da criança ${ }^{(13)}$.

Da mesma forma, a integralidade do cuidado à criança requer a superação do modelo assistencial reducionista, como uma atribuição exclusiva da equipe de saúde, remetendo a um compromisso centrado nas políticas sociais, na participação popular no processo das decisões e controle das condições de saúde do indivíduo e da coletividade, e no compromisso do Estado em proteger a criança ${ }^{(14-15)}$. 
Diante disso, o objetivo deste estudo foi analisar a contraposição entre os discursos do Poder Judiciário e do Poder Executivo, contendores em decisão judicial, acerca do direito à saúde de criança em situação de adoecimento crônico com desnutrição grave.

\section{MÉTODO}

Trata-se de estudo descritivo de abordagem qualitativa, baseado em análise documental, em que são salientados elementos do documento, mediante sua desconstrução minuciosa, buscando-se uma interpretação coerente ${ }^{(16)}$.

$\mathrm{O}$ documento em questão foi uma decisão judicial proferida pelo Tribunal de Justiça do Estado de Mato Grosso (TJMT) versando sobre demanda do direito à saúde de criança de quatro meses, em tratamento de doença inflamatória intestinal crônica e desnutrição grave, com solitação de fórmula láctea e medicamentos. Tal decisão consta do banco de dados da pesquisa matricial, composto por 338 decisões judiciais julgadas em segunda instância referentes ao período de $01 \mathrm{de}$ abril de 2008 a 31 de março de 2009 pelo TJMT. Estas foram analisadas, quantitativamente, em uma primeira fase da pesquisa ${ }^{(7)}$.

Na segunda fase da pesquisa houve uma revisita ao banco de dados da pesquisa matricial, com o foco da busca em demandas realizadas por pessoas com mais de um agravo à saúde, ou seja, experienciando comorbidades. Obteve-se o total de 27 decisões, das quais se buscou identificar elementos comuns em termos de estrutura e composição. Após leitura destas, elegeu-se uma única para submeter à análise, visto ter sido considerada exemplar das demais, no sentido de reiterar os discursos nelas emanados. O critério importante para definir a eleição da demanda, aqui analisada, foi o fato de envolver uma criança, protegida por dispositivo legal específico - o ECA.

Tal decisão foi abordada em sua ementa, relatório e voto do Magistrado e acórdão. Nela buscou-se analisar o discurso do autor, no caso o Estado de Mato Grosso, e o discurso do Poder Judiciário, proferido pelo Relator/ Magistrado. Destes dois discursos destacou-se as expressões significativas que denotavam o "poder de dizer" acerca do direito a saúde frente à demanda pleiteada ${ }^{(17)}$.

Utilizou-se, como matriz analítica dos discursos proferidos pelos contendores, o ECA e as Políticas de Atenção à Saúde da Criança e do Adolescente ${ }^{(11,18)}$. A partir desta análise, elencaram-se três eixos de alegações que foram utilizadas por ambos, um para afirmar, outro para refutar o direito pleiteado. Observa-se que os argumentos, em torno dos quais eles se embatem, têm como base, geralmente, os mesmos dispositivos legais.

Manteve-se a transcrição literal de trechos do referido documento como elementos de análise, tanto do discurso do Magistrado quanto do Estado. Nos trechos transcritos utilizou-se da sigla 'DJ' para remeter a decisão Judicial analisada e trechos dos discursos nela contidos, enumerados segundo a folha do documento em que se encontra. As siglas 'DM' e 'DE' foram utilizadas para designar, respectivamente, o Discurso do Magistrado e o Discurso do Estado na decisão.

Embora para este estudo específico o corpus analisado seja de domínio público, os nomes das pessoas citadas no documento foram mantidos em sigilo em respeito ao princípio do anonimato. Destaca-se também que a pesquisa matricial foi aprovada pelo Comitê de Ética em Pesquisa do Hospital Universitário Julio Muller, sob n. 671/CEP-HUJM/09.

\section{RESULTADOS}

A decisão judicial em análise foi julgada em segunda instância no TJMT. O Estado de Mato Grosso no documento analisado é tomado como "Autor", visto que recorreu da decisão desfavorável anteriormente proferida em primeira instância, na qual foi réu. Desse modo, tratou-se de um recurso de Agravo de Instrumento decisão judicial ocorrida em segundo grau, ou seja, o Estado recorreu da decisão do magistrado com objetivo de contrapor uma determinação judicial dada na primeira instância - tramitada pelo agravante em face da "Obrigação de Fazer" negada em primeira instância.

Essa decisão foi analisada em segunda instância pela comarca do Município de Sinop, localizado no interior do Estado, e trouxe como agravados o Estado de Mato Grosso e o próprio Município; e tem como agravante "um bebê de apenas quatro meses", representado por seu pai, que demandou "fornecimento gratuito de fórmula láctea isenta de lactose e os medicamentos Prednisona, Sulfazalacina, Protovit, Luftal, Ranitidina, Bactrim, Florax, Profol" (Decisão Judicial, fl.03-DM). Esses objetos da demanda se destinavam ao tratamento de doença inflamatória intestinal crônica e desnutrição grave.

Este pedido foi negado pelo Estado de Mato Grosso (MT) e pelo Município de Sinop. O Estado utilizou-se de argumentos diversos para sustentar tal negativa; estes foram agrupados em três eixos de alegações: a) a necessidade de gestão, pelo Estado, na distribuição de medicamentos; b) o desconhecimento do Estado sobre 
o risco de morte do bebê; c) a limitação dos recursos financeiros em saúde.

Relativo à necessidade de gestão, pelo Estado, na distribuição de medicamentos, este assevera que deve realizar o atendimento às necessidades dos cidadãos de forma organizada:

Sustenta que para atendimento da necessidade da população, a Secretaria de Estado de Saúde criou o Protocolo Clínico e Diretrizes Terapêuticas com a função de estabelecer os critérios de diagnóstico de cada doença com o medicamento disponivel, acompanhamento e verificação de resultados, racionalização, prescrição e fornecimento de medicamentos, equipamentos e tratamentos dispensáveis. (Decisão Judicial, fl.04-DE)

O Magistrado, frente a esta negação do Estado, argumenta que, independentemente de protocolos e/ou diretrizes terapêuticas, é dever Constitucional do Estado garantir o acesso da população à saúde e, neste caso específico, por tratar-se de um bebê que se encontra salvaguardado pelo ECA, o qual dispõe de direitos específicos a crianças e adolescentes:

[...] um bebê, cujos cuidados certamente devem ser redobrados e cuja proteção encontra-se resguardada, inclusive, pelo Estatuto da Criança e do Adolescente que lhe garante direito à proteção integral e atendimento preferencial. (Decisão Judicial, fl.08-DM)

Quanto ao desconhecimento do risco de morte do bebê, o Estado argumenta que não havia tal informação, de forma explícita, nos relatórios médicos. Conforme trecho:

Alega que o fundamento para indeferir a medida requerida de que nos relatórios médicos não consta, expressamente, existência de risco de morte ao agravante. (Decisão Judicial, fl.03-DE)

Sobre este argumento o relator contradiz o Estado nos seguintes termos:

Neste diapasão tenho que o fato de não constar no relatório médico, expressamente, que o Agravante está em risco de morte, não exclui a obrigação do Estado quanto ao fornecimento dos remédios buscados, uma vez que resta evidenciada a necessidade de fazer uso da fórmula láctea isenta de lactose, como também, dos medicamentos elencados na inicial, em razão da sua frágil condição de saúde, especialmente, levando em consideração tratar-se de um bebê [...]. (Decisão Judicial, fl.08-DM)

O último argumento do Estado é de que não cumpre a decisão por falta de ressarcimento e pelo temor da retirada de recursos de todo um sistema em benefício de "apenas" uma pessoa. Assim, o Estado utilizou o discurso comum do direito coletivo contraposto ao direito individual:

Afirma que não quer eximir-se da sua responsabilidade de prestar medicamentos ao Agravante, mas apenas evitar que os recursos orçamentários destinados ao atendimento de todo o sistema de saúde sejam desviados para atender a interesses em contrapartida de ressarcimento pela União ou pelo Municipio. (Decisão Judicial, fl.04-DE)

Sobre esta colocação, o Magistrado rebate no seguinte trecho:

[...] o Estado deve imprimir esforços no sentido de viabilizar o acesso e a prestação à saúde, indispensável para que a pessoa viva com dignidade, sendo certo que eventuais dificuldades financeiras enfrentadas pelo ente Público não podem servir de obstáculos para justificar o abandono e desamparo da população, especialmente, daqueles que necessitam de medicamentos gratuitamente e que no caso, são essenciais à saúde do agravante. (Decisão Judicial, fl.06-DJ)

\section{DISCUSSÃO}

O discurso do Poder Judiciário, em sua totalidade, se firmou na Constituição Federal a fim de se fazer cumprir a decisão, alegando que é o direito à vida e à saúde que está em pauta, e que o Estado não pode medir esforços para cumprir tais direitos. Para tanto, os profissionais do direito se utilizaram da jurisprudência ${ }^{(19)}$, como conjunto de decisões dos tribunais que representa a visão daquele Tribunal no determinado momento sobre as questões levadas a julgamento.

No que se refere ao primeiro argumento, que trata da necessidade de gestão, pelo Estado, na distribuição de medicamentos, o Estado de MT afirma o fato dos medicamentos demandados judicialmente pelo agravante não estarem presentes em portarias, se referindo 
a um protocolo, o qual exige que tais medicamentos devam estar cadastrados na Agência Nacional de Vigilância Sanitária (ANVISA) ou constar na RENAME. Tais medicamentos são assegurados pelo MS por serem considerados básicos e indispensáveis para atender a maioria dos problemas de saúde da população ${ }^{(3)}$.

Muitos medicamentos prescritos em demandas judiciais não constam em portarias do governo por ainda não terem sua eficácia comprovada, mas são prescritos por preferência médica, ou por influência da indústria farmacêutica ${ }^{(20)}$. No entanto, nem todos os medicamentos pleiteados judicialmente são de alto custo, ou são novos no mercado; pelo contrário, muitos compõem a farmácia básica e figuram nas listas e protocolos do SUS ${ }^{(21)}$.

Estudo sobre ações judiciais solicitando medicamentos à Secretaria do Estado de Saúde de São Paulo, em 2005, mostrou que a maioria (73\%) dos fármacos pleiteados possuíam a mesma fórmula dos cadastrados na Relação Municipal de Medicamentos Essenciais (REMU$M E)^{(21)}$. Também estudo sobre autorizações de compra de medicamentos pela Secretaria do Estado de Saúde de Santa Catarina, para atender solicitações judiciais ou administrativas, constatou que $32,2 \%$ dos medicamentos demandados judicialmente eram padronizados pelo SUS ${ }^{(20)}$.

$\mathrm{Na}$ decisão aqui analisada, a RENAME de 2007, em vigor à época, trazia em sua relação os medicamentos Bactrim, Prednisona, Ranitidina e Sulfazalacina, embora algumas aí constando com o nome comercial. A Sulfazalazina, nome do princípio ativo da medicação Sulfazalacina, está especificada nesta RENAME como sendo de uso na doença inflamatória intestinal, agravo apresentado pela criança demandante na decisão. Portanto, entre os medicamentos solicitados para esta, a maioria estava contemplada na RENAME, e todos constavam na lista da ANVISA ${ }^{(4)}$.

A prescrição e fornecimento de medicamentos e equipamentos e os tratamentos dispensáveis foram regulamentados pelo Ministério da Saúde por meio do Protocolo Clínico e Diretrizes Terapêuticas que estabelece tais critérios, dentre outros, para cada doença ${ }^{(3)}$. Estudos evidenciam que, na prática, tal regulamentação do acesso tem se mostrado demasiadamente burocrática, tornando a busca pelo medicamento uma verdadeira peregrinação para as pessoas ${ }^{(8-10)}$.

O tratamento medicamentoso dos agravos crônicos, tal como a doença inflamatória intestinal crônica e a desnutrição grave, torna-se inacessível para a maioria da população, quer pelo alto custo de cada medicamento, quer pelo custo total do tratamento que, muitas vezes, perdura por toda a vida ${ }^{(3)}$. Acresce-se a isso que a cronicidade do agravo apresentado pela criança em questão a acompanhará por toda a vida, o que torna a situação potencialmente mais complexa, acarretando múltiplos custos para ela e sua família; custos, estes, dificilmente quantificáveis e previsíveis ${ }^{(22-23)}$.

Em relação ao segundo argumento do desconhecimento do risco de morte da criança pelo Estado de MT, pode-se afirmar que este discurso é incongruente com as políticas de proteção de crianças e adolescentes existentes, as quais estabelecem a responsabilidade das Secretarias Municipais de Saúde no acompanhamento da saúde e do crescimento das crianças, devido sua significativa vulnerabilidade e altas taxas de mortalidade que ainda recai sobre essa população. Tais políticas de cuidado infantil preceituam linhas de cuidado integral contínuo entre estas, a que incentiva e qualifica o acompanhamento do crescimento e desenvolvimento ${ }^{(12,14,23)}$.

Diante disto, torna-se duplamente inconcebível o argumento do Estado de desconhecer a condição de um bebê de nove meses com desnutrição grave por doença inflamatória intestinal crônica, considerando que este tem o dever de acompanhar esta criança, direito este garantido pelo ECA. Em tal dispositivo consta o direito incondicional a vida e a saúde, mediante a efetivação de políticas públicas; e seu acompanhamento de saúde está claramente estabelecido como prioritário em todos os níveis de atenção à saúde ${ }^{(11)}$.

O Magistrado reforça o caráter inquestionável do direito à vida e à saúde, argumentando tratar-se de criança que precisa de fórmula láctea específica que visa garantir a nutrição necessária e, consequentemente, promover seu crescimento e desenvolvimento saudável. Também os medicamentos, para controle do seu agravo, são considerados, pelo Magistrado, como necessários para seu tratamento, dada sua condição frágil de saúde. Desta forma, a obrigação do Estado seria de impedir ou minimizar os danos e riscos a sua vida.

Percebe-se que as alegações feitas pelo Estado são bastante preocupantes suscitando questionamentos quanto à efetividade das políticas que tenham como foco criança ou adolescente, dada à dupla omissão nos seus deveres: o de conhecer as necessidades de saúde de um lactente de quatro meses de vida desnutrido e o de sustentar o seu tratamento. Desta forma, não poderia o Estado se abster de proporcionar a fórmula láctea e os medicamentos necessários ao tratamento da criança, alegando não conhecer suas necessidades de saúde, visto que é o próprio Estado que institui políticas para cuidados específicos à criança e se torna o principal 
ordenador das ações necessárias para sua efetivação ${ }^{(2)}$.

Sobre o terceiro argumento de limitação dos recursos financeiros em saúde, o Magistrado afirma que o Estado não pode alegar dificuldade financeira para negar medicação a um menor impúbere sob a justificativa de que seriam desviados recursos destinados ao atendimento de todo o serviço de saúde. A criança em pauta, além de estar protegida constitucionalmente pelo ECA, também usufrui dos direitos de todo o cidadão: a proteção a vida e a saúde. Então, ao não fornecer o medicamento pedido, o Estado fere os dizeres da Carta Magna ${ }^{(2)}$.

O direito constitucional à saúde, no que se refere ao tratamento medicamentoso, como no caso em tela, deve ser garantido pelo Estado na forma da regularidade no seu fornecimento, bem como do seu acesso universal, efetivando a equidade e integralidade preceituadas no artigo 196 da Constituição. Assim, a discussão acerca do direito individual em detrimento do direito coletivo é um desafio ao SUS que, se por um lado deve garantir o direito fundamental a todos, por outro deve garantir o direito individual de cada um. Considera-se que a questão central neste contexto é a legitimidade da norma que restringe a liberdade individual e, deste modo, correlacionada com a ideia do que se entende por lei justa ${ }^{(20)}$.

Estudos que versam sobre a judicialização da saúde têm mostrado que uma das principais justificativas do Estado é que este tipo de intervenção no SUS aprofundaria as iniquidades no acesso à saúde, privilegiando determinados segmentos e indivíduos com maior poder de reivindicação, em detrimento de outros, na medida em que necessidades individuais ou de grupos determinados seriam atendidas, em prejuízo às necessidades de outros grupos e indivíduos ${ }^{(6,21)}$. Mas, se para o SUS, todo cidadão é igual perante a lei e deve ser atendido de acordo com suas necessidades ${ }^{(8)}$, a equidade se refere à diminuição das diferenças que podem ser evitadas no contexto de atendimento à saúde.

Por fim, a posição do Magistrado em prover a demanda com base no agravo da criança, frente a qual ela se encontra vulnerável, reflete a busca por priorizar o atendimento aos mais desamparados, atitude baseada no princípio de equidade ${ }^{(9)}$.

Neste caso, a situação reveste-se de outras implicações, necessitando ainda maior atenção por se tratar de criança de quatro meses, com intolerância a lactose e já em desnutrição grave, o que a torna duplamente vulnerável - por ser lactente e por apresentar-se desnutrida. Essa vulnerabilidade deveria ser o foco principal da preocupação do Estado, pois a intolerância à lactose, embora possa ocorrer em qualquer idade, é mais comum após os cinco anos de idade, sendo rara sua manifestação desde o nascimento ${ }^{(18,23)}$. Assim, ações prioritárias das políticas de saúde pelo Estado deveriam ter sido desenvolvidas no sentido de proteger esta criança de consequências mais graves a sua saúde.

\section{CONCLUSÃo}

Os discursos analisados proferidos pelo Poder Judiciário e Poder Executivo, com relação ao direito a saúde de um lactente em risco de morte por desnutrição grave decorrente de intolerância a lactose, evidenciam a contradição do discurso do Estado brasileiro na proteção de seus cidadãos, especificamente as crianças.

Esta contradição mostra que o Estado descumpre abertamente a Constituição Federal e o ECA, considerando as questões administrativas e econômicas como mais prioritárias que os direitos à vida e ao bem estar de uma criança vulnerável. Além disso, estes resultados demonstram a influência das políticas neoliberais que predicam a diminuição da presença estatal na vida das pessoas, por meio de uma política econômica que aumenta as iniquidades sociais.

Este estudo apontou que as políticas de saúde ainda não permitem a implementação efetiva da organização das práticas de saúde, e de um modelo assistencial que preconize a integralidade e a equidade na atenção às crianças portadoras de condições crônicas de adoecimento.

Destarte, estes resultados permitem uma reflexão sobre possibilidades do campo jurídico e da saúde serem complementares na garantia do direito à saúde de todo cidadão brasileiro, através de atuação sinérgica em seus esforços e não de oposição. Mostra, portanto, caminho fértil para outros estudos que possam ter por foco o fenômeno da judicialização da saúde, não apenas em seu dimensionamento quantitativo, mas em suas implicações para as práticas profissionais de gestão e de atenção à saúde.

\section{REFERÊNCIAS}

1. Brasil. Constituição da República Federativa do Brasil. Brasília: Senado; 1988.

2. Ministério da Saúde (BR). Lei n. 8080, sancionada em 19 de Setembro de 1990, a qual dispõe sobre as condições para a promoção, proteção e recuperação da saúde, a organização e o funcionamento dos serviços correspondentes e dá outras providências. Diário Oficial da União, Brasília, 19 set 1990.

3. Ministério da Saúde(BR). Secretaria de Políticas de Saúde.

Cogitare Enferm. 2013 Out/Dez; 18(4):647-54 
Departamento de Atenção Básica. Política nacional de medicamentos. Brasília: Ministério da Saúde; 2001.

4. Souza RR. O Programa de Medicamentos Excepcionais: protocolos clínicos e diretrizes terapêuticas: medicamentos excepcionais. Brasília: Ministério da Saúde/Secretaria de Assistência à Saúde; 2002.

5. Ministério da Saúde (BR). Secretaria de Atenção à Saúde. Departamento de Atenção Básica. Política Nacional de Alimentação e Nutrição, [Internet] 2011 [acesso em 25 mar 2013]. Disponível: http://89.28.128.100/nutricao/ docs/geral/pnan2011.pdf.

6. Chieffi AL, Barata RB. Judicialização da política pública de assistência farmacêutica e eqüidade. Cad. Saúde Pública. [Internet] 2009;25(8) [acesso em 05 out 2010]. Disponível: http://dx.doi.org/10.1590/S0102311X2009000800020

7. Bellato R, Araújo LFS, Nepomuceno MAS, Mufato LF, Corrêa GHLZT. Mediação do direito à saúde pelo tribunal de justiça: análise da demanda. Texto Contexto Enferm. [Internet] 2012;21(2) [acesso em 20 abr 2013]. Disponível: http://dx.doi.org/10.1590/S0104-07072012000200013

8. Petean E, Araújo LFS, Bellato R, Wunsch CG, Mufato LF, Nepomuceno MAS. Direito à saúde: demanda por suplementos no Tribunal de Justiça. Rev Eletr Enf. [Internet]2012;4(1) [acesso 05 dez 2012]. Disponível:http:// www.fen.ufg.br/fen_revista/v14/n1/pdf/v14n1a08.pdf

9. Soares JL, Araújo LFS, Bellato R, Corrêa GHLZT, Mufato LF, Nepomuceno MAS. Demanda por cirurgias mediadas pelo poder judiciário: considerações sobre o direito à saúde. Rev. baiana de saúde pública. [Internet] 2011;35(4) [acesso em 09 jun 2012]. Disponível: http://inseer.ibict. br/rbsp/index.php/rbsp/article/viewFile/262/pdf_75

10. Souza IP, Bellato R, Araújo LFS, Almeida KB, Nepomuceno MAS, Mufato LF. Direito à saúde no Tribunal de Justiça: demanda por medicações em oncologia. Rev Rene. 2012;13(4):919-28.

11. Brasil. Lei n. 8.069 de 13 de julho de 1990. Dispõe sobre a proteção integral à criança e ao adolescente. Brasília: Diário Oficial da União, 13 jul, 1990.

12. Chiesa AM. Autonomia e resiliência: categorias para o fortalecimento da intervenção na atenção básica na perspectiva da promoção da saúde [tese]. São Paulo (SP): Universidade de São Paulo; 2005.

13. Sousa FGM, Erdmann AL, Mochel EG. Modelando a integralidade do cuidado à criança na Atenção Básica de Saúde. Rev. Gaúcha Enferm. [Internet] 2010;31(4) [acesso $05 \mathrm{dez}$ 2012]. Disponível: http://dx.doi. org/10.1590/S1983-14472010000400013

14. Prado SRLA, Fujimori E, Cianciarullo TI. A prática da integralidade em modelos assistenciais distintos: estudo de caso a partir da saúde da criança. Texto Contexto Enferm. [Internet] 2007;16(3) [acesso $05 \mathrm{dez}$ 2012]. Disponível: http://www.scielo.br/pdf/tce/v16n3/ a04v16n3.pdf

15. Meirelles BHS, Erdmann AL. A interdisciplinaridade como construção do conhecimento em saúde e enfermagem. Texto Contexto Enferm. [Internet] 2005;14(3) [acesso $05 \mathrm{dez}$ 2012]. Disponível: http:// dx.doi.org/10.1590/S0104-07072005000300013

16. Cellard A. A análise documental. In: Poupart J, Deslauriers JP, Groulx LH, Laperrière A, Mayer R, Pires AP. A pesquisa qualitativa: enfoques epistemológicos e metodológicos. Petrópolis: Vozes; 2008. p. 295-316.

17. Minayo MCS. Contradições e consensos na combinação de métodos quantitativos e qualitativos. São Paulo: Hucitec; 2008.

18. Gaíva MAM, Monteschio CAC. Políticas de atenção à saúde da criança e do adolescente - implicações para a atuação do enfermeiro. Proenf: Saúde da Criança e do Adolescente. 2013; 7(4):9-46.

19. Tribunal Superior Eleitoral (BR). Pesquisa de Jurisprudência - Perguntas frequentes, [Internet] 8 de junho de 2012 [acesso em 12 mar 2013]. Disponível: http://www.tse.jus.br/jurisprudencia/pesquisa-dejurisprudencia/perguntas-frequentes

20. Leite SN, Pereira SMP, SilvaP, Nascimento Jr. JM, Cordeiro BC, Veber AP. Ações judiciais e demandas administrativas na garantia do direito de acesso a medicamentos em Florianópolis-SC. Rev. Direito Sanit. [Internet] 2009;10(2) [acesso em 12 mar 2013]. Disponível: http:// www.revistas.usp.br/rdisan/article/view/13161/14968

21. Vieira FS, Zucchi P. Distorções causadas pelas ações judiciais à política de medicamentos no Brasil. Rev. Saúde Públ. [Internet] 2007;41(2) [acesso em 12 mar 2013]. Disponível: http://dx.doi.org/10.1590/S003489102007000200007

22. Hiller M, Bellato R, Araújo LFS. Cuidado familiar à idosa em condição crônica por sofrimento psíquico. Esc. Anna Nery. [Internet] 2011;15(3) [acesso em 12 mar 2013]. Disponível: http://dx.doi.org/10.1590/S141481452011000300015 
23. NASPGHAN - Sociedade Norte-americana de Gastroenterologia Pediátrica, Hepatologia e Nutrição. Intolerância à lactose em crianças. Informe [Internet] 2010 [acesso 07 mai 2012]. Disponível: http://www. naspghan.org/userassets/Documents/pdf/diseaseInfo/ Lactose-P.pdf 